\title{
Damage Detection in Aeronautical Profile by using Frequency Changes and Optimization Algorithms
}

\author{
Guilherme F. Gomes ${ }^{a 1}$, Sebastião S. Cunha Jr ${ }^{a}$ and Antonio C. Ancelotti Jr. ${ }^{a}$ \\ ${ }^{a}$ Mechanical Engineering Institute, Federal University of Itajubá, Itajubá, MG, Brazil \\ Received on December 13, 2015 / accepted on May 12, 2016
}

\begin{abstract}
The structural health monitoring (SHM) by damage detection studies is present in many engineering applications because they enable that correctives actions be applied to make sure the structural safety. This work has developed a numerical study to assess damage detection in NACA airfoils, because most non-destructive inspection techniques used in the aeronautical field need a high level of operator experience to its successful completion and later interpretation of results. This work is based on the resolution of the direct and inverse problem by combining data from a simulated model and experimental data. A local stiffness reduction is applied in a specific element as the damaged model and Genetic Algorithm is used to solve a global optimization problem considering natural frequencies. The effect of the two damage variables (damage location and severity) are studied and results of as shown a good performance to detect and identify the structural damage also with noisy data.
\end{abstract}

Keywords: Damage detection, genetic algorithm, NACA 4412, vibrations, composite materials.

\section{Introduction}

Fiber-reinforced composite materials possess advantages of low weight, high stiffness, high strength, and fatigue resistance compared to metallic alloys, and they may be more extensively used in future for primary components of aircraft structures. However, fiber-reinforced composites have several disadvantages. For example, it is more possible to produce damage in their manufacturing and usage processes. Failures and damage of composite structures, especially aircraft structures, may cause tragic consequences. Furthermore, the failure mechanisms of composites are much more complicated than that of conventional materials, and the increasing use of composite materials in engineering structures has brought many new problems for maintenance. Therefore, fiber-reinforced composites' damage detection has become a very important issue ([1]).

Aerospace structures are very sensitive to damage since it can lead to major failure. Therefore daily costly inspections are a part of regular maintenance procedures. Nowadays commercial and military aircrafts are increasingly using composite materials to take advantage of their excellent specific strength and stiffness properties but impacts on composites due to bird-strike, hail-storm cause barely visible impact damage (BVID) that underscores the need for robust SHM methods ([3]).

The flight environment is very harsh due to large changes in humidity, temperature, pressure, speed, and loading conditions. These effects cause a lot of stress to the aircraft frame. As a result, corrosion, delamination, cracks, disbonds, and other failures creep in once the aircraft is in service for some time. Traditionally, visual inspection, accompanied by ultrasound bulk wave or eddy current technology, is often used to obtain general information on the structural health conditions. However, the inspection is limited

\footnotetext{
${ }^{1}$ E-mail Corresponding Author: guilhermefergom@unifei.edu.br
} 
to a point-bypoint manner and is very time consuming. In most cases, erection of scaffolding or disassembly of the structure is needed to inspect the interior and inaccessible components, being very labour intensive and possibly also resulting in maintenance-induced damages. The development of a general, in situ health monitoring system that can inspect a relatively large area, instantaneously provide reliable and quantitative structural health data such as defect type, location and severity level, minimize and eventually eliminate the need for structural disassembly $([2])$.

It is essential to ensure the safety and reliability of in-service structures by detecting structural cracking, corrosion, delamination, material degradation and other types of damage in time. Utilization of artificial intelligence network system can enable automatic inspection of such damages ultimately. Good results were obtained for simulated defects on the wing panel. The proposed method of using both modal data and optimization method is very useful for identifying damage in large-scale in aeronautical structures. structures.

\section{Problem Formulation}

The modeling of the damage detection problem addressed in this paper was formulated on two fronts: modelling the direct problem, modelled by the finite element method and the inverse problem using optimization method for the search of variables for the identification of structural damage location and severity.

\subsection{Direct Problem: Finite Element Model}

The demand for improved wings for early air-planes and the lack of any generally accepted wing theory led to tests of large numbers of wings with shapes gradually improving as the result of experience. The NACA investigations were further systematized by separation of the effects of camber and thickness distribution, and the experimental work was performed at higher Reynolds numbers than were generally obtained elsewhere. The wing sections now in common use are either NACA sections or have been strongly influenced by the NACA investigations. For this reason, and because the NACA sections form consistent families, detailed attention will be given only to modern NACA wing sections ([5]). According to to the author, the cambered wing sections of all NACA families of wing sections considered here are obtained by combining a mean line and a thickness distribution. The process for combining a mean line and a thickness distribution to obtain the desired cambered wing section is illustrated in Fig.1. The leading and trailing edges are defined as the forward and rearward extremities, respectively, of the mean line. The chord line is defined as the straight line connecting the leading and trailing edges. Ordinates of the cambered wing sections are obtained by laying off the thickness distributions perpendicular to the mean lines. The abscissas, ordinates, and slopes of the mean line are designated as $x_{c}, y_{c}$, and $\tan (\theta)$,respectively. If $x_{u}$ and $y_{u}$ represent, respectively, the abscissa and ordinate of a typical point of the upper surface of the wing section and $y_{t}$ is the ordinate of the symmetrical thickness distribution at chord wise position $x$, the upper-surface coordinates are given by the following relations:

$$
\begin{gathered}
x_{u}=x-y_{t} \sin \theta \\
y_{u}=y_{c}+y_{t} \cos \theta
\end{gathered}
$$

The corresponding expressions for the lower-surface coordinates are

$$
\begin{gathered}
x_{l}=x+y_{t} \sin \theta \\
y_{l}=y_{c}-y_{t} \cos \theta
\end{gathered}
$$

The center for the leading-edge radius is found by drawing a line through the end of the chord at the leading edge with a slope equal to the slope of the mean line at that point and laying off a distance from the leading edge along this line equal to the leading-edge radius. This method of construction causes the cambered wing sections to project slightly forward of the leading-edge point. Because the slope at the leading edge is theoretically infinite for the mean lines having a theoretically finite load at the leading edge, the slope of the radius through the end of the chord for such mean lines is usually taken as the slope of 
the mean line at $x / c$ equals 0.005 . This procedure is justified by the manner in which the slope increases to the theoretically infinite value as $x / c$ approaches $\mathrm{O}$. The slope increases slowly until very small values of $x / c$ are reached. Large values of the slope are thus limited to values of $x / c$ very close to 0 and may be neglected in practical wing-section design ([5]). When the NACA four-digit wing sections were derived, it was found that the thickness distributions of efficient wing sections such as the Göttingen 398 and the Clark $\mathrm{Y}$ were nearly the same when their camber was removed (mean line straightened) and they were reduced to the same maximum thickness. The transversal profile of the NACA four digity is shown in Figure 1. The thickness distribution for the NACA four-digit sections was selected to correspond closely to that for these wing sections and is given by the following equation:

$$
\pm y_{t}=\frac{t}{0.20}\left(0.29690 \sqrt{x}-0.12600 x-0.35160 x^{2}+0.28439 x^{3}-0.10150 x^{4}\right)
$$

where $t=$ maximum thickness expressed as a fraction of the chord. The leading-edge radius is:

$$
r_{t}=1.1019 t^{2}
$$

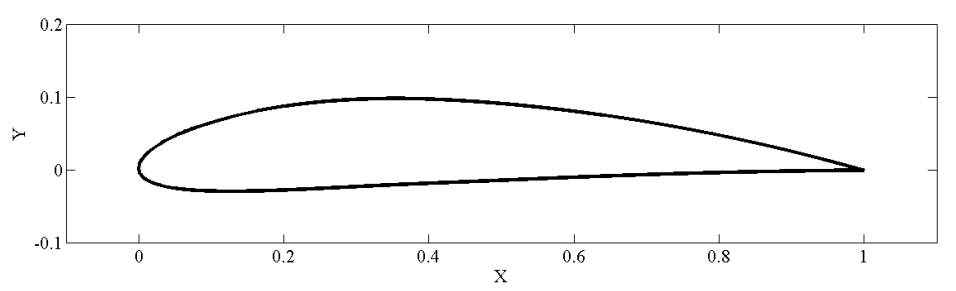

Figure 1: NACA 4412 profile.

Then, the direct problem formulation was carried out with the aid of the finite element software ANSYS. For this, the use of shell elements was employed with 6 degrees of freedom (three rotation and translation 3 of the nodes) . A structured mesh were sufficient to obtain reliable results and lower computational cost . The structure was discretized into 20x10 elements. The properties of the material used in the modeling are shown in Table 1, with data for the carbon/epoxy material.

Table 1: Material Properties.

\begin{tabular}{cc}
\hline Property & Value \\
\hline$E_{1}$ & $83.02 \mathrm{MPa}$ \\
$E_{2}$ & $5.13 \mathrm{MPa}$ \\
$G_{12}$ & $8.37 \mathrm{MPa}$ \\
$\nu 12$ & 0.32 \\
$\rho$ & $1408.1 \mathrm{~kg} / \mathrm{m}^{3}$ \\
\hline
\end{tabular}

\subsubsection{Damage Model}

According to [4], for a multi-degree-of-freedom undamped linear dynamic system, the equation of motion is:

$$
M \ddot{y}(t)+K y(t)=Y(t)
$$

where $M$ is the system mass matrix; and $K$, the system stiffness matrix with initial conditions $\ddot{y}(t)=y(t)=0$. Both mass and stiffness matrices are of the order $(n \times n) . y(t)$ and $Y(t)$ are the physical displacement and applied load vectors of order $(n \times 1)$ respectively, where $n$ is the number of degrees of freedom. The associated $j$ th eigenvalue equation is: 


$$
K \phi_{j}-\lambda_{j} M \phi_{j}=0 \text { for } j=1, \ldots, m_{u}
$$

where $\phi_{j}$ is eigenvector (mode shape) and $\lambda_{j}$ the eigenvalue (natural frequency) of the structure; and $m_{u}$ the total number of mode shapes obtained for the undamaged structure. In the finite element model of the structure, the global stiffness matrix can be represented as an assemblage of element stiffness matrices, i.e.:

$$
J=\sum_{i=1}^{m} k_{i}
$$

where $k_{i}$ represents the stiffness matrix of the $i$ th element; $m$ the total number of elements and $\sum$ represents the assembly of elemental stiffness matrices based on nodal connectivity and the associated degrees of freedom.

Structural damage often causes a loss of stiffness in one or more elements of a structure, but not a loss in the mass. In the theoretical development that follows, the NACA profile is modeled in an finite element analysis (FEM), and damage is assumed to affect only the stiffness matrix of the system. Small changes in the stiffness of the system produce small changes in the square of the modal frequencies $\lambda_{i}$ and the mode shapes $\phi_{i}$. When damage occurs in the structure, it can be represented as a small perturbation in the original system. Thus, the stiffness matrix $K_{d}$, the $i$ th modal eigenvalue $\lambda_{i}^{d}$, and the $i$ th mode shape $\phi_{i}^{d}$ of the damaged system can be expressed as:

$$
\begin{gathered}
K^{d}=K \sum_{j=1}^{m}{ }_{j}=\sum_{j=1}^{m} \beta_{j} k_{j} \\
\lambda_{i}^{d}=\lambda_{i}+\Delta \lambda_{i} \\
\phi_{i}^{d}=\phi_{i}+\Delta \phi_{i}
\end{gathered}
$$

Thus, the stiffness matrix of the damaged structure $(K d)$ can be represented as an assemblage of element stiffness matrices multiplied by reduction factors $\beta_{j}(j=1,2, \ldots, m)$ associated with each of the " $M$ " elements, where superscript $d$ denotes the damaged case. The Fig. 2 show the meshed NACA airfoil with a localized structural damage modeled as a stiffness reduction at the element number 230 .

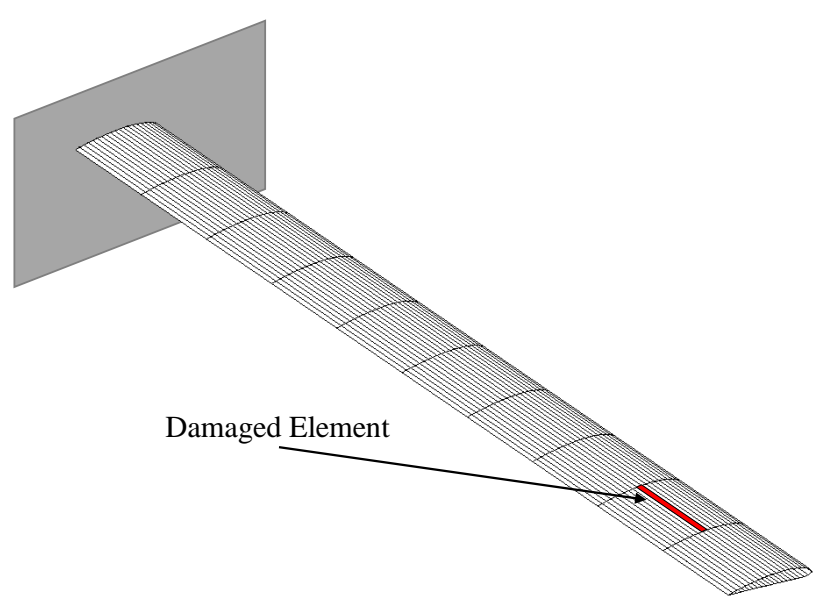

Figure 2: The finite element model of the laminated plate and the damaged element number 230 . 


\subsection{Inverse Problem: Optimization Techniques}

\subsubsection{Genetic Algorithm (GA)}

Based on the concept of genetics, GA simulates the evolutionary process numerically. Analogous to gene in genetics, GA represents the parameters in a given problem by encoding them in a string. Instead of finding the optimum from a single point in traditional mathematical optimization methods, in GA a set of points that is, a population of coded strings, is used to search for the optimal solution, Simple GA consists of three basic operators: reproduction, crossover and mutation ([7]).

The GA procedure uses random initial populations and through selection, crossover and mutation in GA, better offspring are fed into the surrogate model for the next iteration. This iterative procedure continues until convergence to the set precision criteria is achieved or the number of generations reaches the maximum set value. The parameter settings used for the GA are listed in Table 2. Every solution in the population is called as an individual and every individual is represented as a chromosome for making it suitable for the genetic operations. The user defined value is used as the initial population of meta heuristic which encode candidate solutions to an optimization problem and evolves better solutions ([8]).

Table 2: Genetic Operators.

\begin{tabular}{cc}
\hline Genetic Operator & Value \\
\hline Population size & 40 \\
Crossover & $60 \%$ \\
Mutation & $2 \%$ \\
Elitism & 2 \\
Generations & 100 \\
\hline
\end{tabular}

\subsubsection{Ant Colony Optimization (ACO)}

The Ant System algorithm is a population-based metaheuristic inspired by the foraging behavior of ants. In nature, many species of ants are almost completely blind and the communication among them is performed through a chemical substance called pheromone. When walking, the pheromone is deposited by the ants on the performed paths, forming trails of pheromone. The following ants by perceiving the pheromone presence, choose the trail with higher concentration of it. These trails help population members to find food sources, as well as the way back. This makes the ants able to find the shortest path between their nest and the food source $([10])$.

The ACO search procedure, the size of the archive, the number of ants in each iteration and the pheromone evaporation rate play an important role in convergence speed of the algorithm. After some trials the constant parameters of the proposed algorithm have been set up as Table 3. The initial archive solution is randomly generated within the specified parameter range; no good guess of initial parameter values is needed.

Table 3: AC Operators.

\begin{tabular}{cc}
\hline Ant Operator & Value \\
\hline Number of Ants & 40 \\
Evaporation Rate & 1.25 \\
Iterations & 100 \\
\hline
\end{tabular}

The main goal of the optimization procedure in this work is to adjust the fractional order $\alpha$ and the damaged element number $N_{\text {elem }}$ in order to obtain the best properties of the damage identification algorithm. Hence, the optimization problem can be written as follows:

$$
\text { Minimize } J=\sqrt{\frac{1}{n} \sum_{i=1}^{n}\left(1-\frac{\omega_{i}^{\text {damaged }}}{\omega_{i}^{\text {calculated }}}\right)^{2}}
$$




$$
\text { Subject to } 1<N_{\text {elem }}<100 \text { and } 0<\alpha<1
$$

where $\omega_{i}^{\text {damaged }}$ is the target frequency shift obtained either from measurements (in the case of experimental validation) or from $\mathrm{FE}$ test cases (numerical validation) and $\omega_{i}^{\text {calculated }}$ is frequency shift obtained from the surrogate model.

A mixed integer programming problem is an optimization problem, linear or nonlinear, with or without constraints, in which some or all decision variables are restricted to have integer values. Such problems frequently arise in various application fields such as process industry, finance, engineering design, management science, process flow sheets, portfolio selection, batch processing in chemical engineering, and optimal design of gas and water distribution networks $([6])$. The variable of the damage position $\left(N_{\text {elem }}\right)$ was optimized based on integer values, i.e., $N_{\text {elem }} \in[1,2, \ldots, 100]$

The optimization routine is introduced to make the search for damage to the geometry of the structure, with interaction between the finite element software where the model structure is adapted to receive the design variables and optimization software that will hold a series of calculations in order to generate input data for the finite element software. The flowchart of the Figure 3 shows the steps to be performed during the damage searching. The method for detecting damage in this study meets three of the four criteria proposed by [9], the detection, localization and quantification of damage (damage models studied). No damage criterion was adopted in this study, based on the assumption that there is already a significant structural damage.

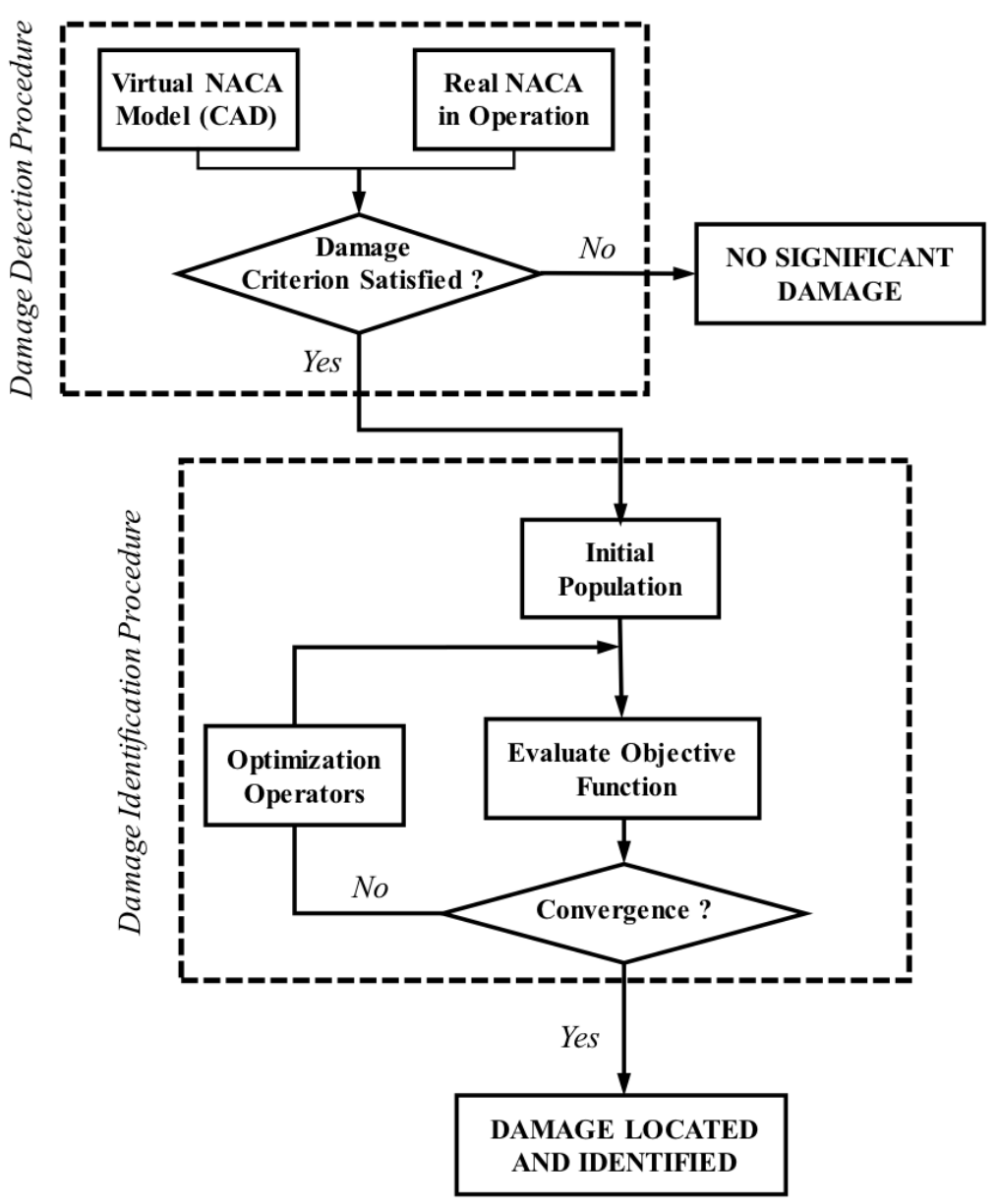

Figure 3: Flowchart of the damage detection procedure. 
Table 4: Natural frequencies of undamaged and damaged profile considering $\alpha=0.1$.

\begin{tabular}{ccccc}
\hline Mode & Undamaged & Damaged & $\left|\omega_{\text {und }}-\omega_{\text {dam }}\right|$ & $\left(\frac{\left|\omega_{\text {und }}-\omega_{\text {dam }}\right|}{\omega_{\text {und }}}\right) \times 100$ \\
\hline 1st & 14.161 & 14.161 & 0.000 & 0.0000 \\
2nd & 71.482 & 71.459 & 0.023 & 0.0322 \\
3rd & 90.588 & 90.580 & 0.008 & 0.0088 \\
4th & 103.710 & 103.680 & 0.030 & 0.0289 \\
5th & 111.750 & 111.730 & 0.020 & 0.0179 \\
6th & 119.380 & 119.200 & 0.180 & 0.1508 \\
7th & 119.990 & 119.840 & 0.150 & 0.1250 \\
8th & 130.060 & 130.030 & 0.030 & 0.0231 \\
9th & 135.240 & 134.930 & 0.310 & 0.2292 \\
10th & 140.500 & 140.460 & 0.040 & 0.0285 \\
\hline
\end{tabular}

\section{Numerical Results}

The initial analysis demonstrates that the stiffness reduction as induced damage changes the dynamic response of the wing. For this a reduction of $80 \%(\alpha=0.20)$ was induced on the structure. The Table 3 show the results and the differences between the natural frequencies of the undamaged and damaged NACA. For some specific modes the reduction is more intensive and selected modes could be a contributory more significantly at the damage detection procedure. Figure 4 shows the first three vibration modes of the wing undamaged and damaged. All those flexural and torsional modes were included at the inverse optimization problem.

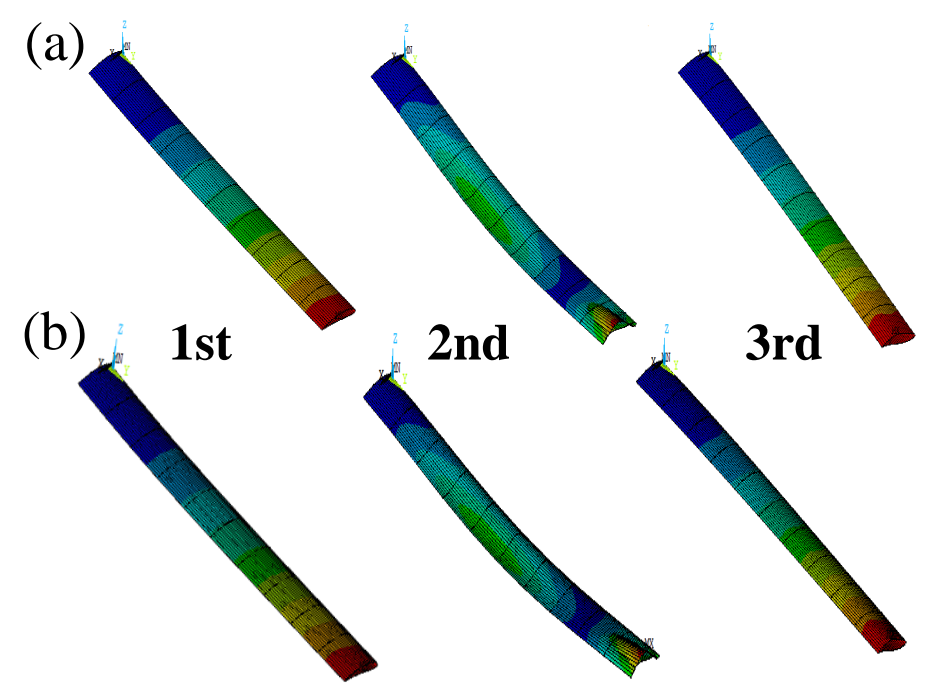

Figure 4: First three mode shapes for the undamaged (up) and damaged NACA airfoil (bottom).

The initial results of the damage detection procedure are shown in the Table 4. A known damage was induced and the GA procedure starts its search for the best value that minimize the objective function showed in the past paragraphs. A mean of five algorithm run was made due to the probabilistic behaviour of heuristic optimization. It is clear that for the numerical case, the results were very satisfactory. The GA found the damage at the same position and severity at the same condition in which it was induced. Results of the damage detection using GA procedure without noisy data are shown in Figure 5.

Complementing the method investigated in this work, the addition of Gaussian white noise (GWN) in the optimization process was considered to verify the robustness of the built method in order to simulate ambient noise coming from a measurement error from sensors or from external environmental. This noise is 
Table 5: Numerical results of damage detection by stiffness reduction.

\begin{tabular}{ccccc}
\hline & \multicolumn{2}{c}{ GA } & \multicolumn{2}{c}{ ACO } \\
\hline & Nelem & $\alpha$ & Nelem & $\alpha$ \\
\hline Objective & 230 & 0.2000 & 230 & 0.2000 \\
Search 1 & 230 & 0.2006 & 231 & 0.2134 \\
Search 2 & 230 & 0.2005 & 231 & 0.2057 \\
Search 3 & 230 & 0.2084 & 230 & 0.1980 \\
Search 4 & 230 & 0.2001 & 231 & 0.2134 \\
Search 5 & 230 & 0.2005 & 230 & 0.2139 \\
Mean & 230 & 0.2020 & 231 & 0.2089 \\
Deviation & 0 & 0.0036 & 0.5477 & 0.0069 \\
Error (\%) & 0 & $1.0100 \%$ & 0.4348 & 4.4400 \\
\hline
\end{tabular}

characterized by values of mean zero and variance $\varepsilon$. Three levels of noise were incorporated in this work: $\varepsilon=1 \%, \varepsilon=5 \%$ and $\varepsilon=10 \%$ where $10 \%$ is a great value for noisy data.

The Tables 5, 6 and 7 summarizes the results obtained from the optimization procedure using GA and ACO. Including 1\% of noise the results (Fig.6) were found in the same surface of the airfoil by using GA, however not so close of the induced damage. The severity $\alpha$ found was almost the same induced $(0.20)$. In other hands, ACO results for the same noise level were worse and the damaged element is far from induced damage, even in the lower surface of the profile. Increase the noise level for $5 \%$, the results were practically the same for GA and ACO. The damaged element obtained by the inverse optimization procedure was situated near of the damaged element induced but on the opposite surface of the wing profile (Fig.7). For high levels of noisy $(10 \%)$ the identified damage were found near of the induced. However, the local damage severity $(\alpha)$ have been found with a great discrepancy among the optimization algorithms studied in this paper. Summarized, in this specific application, GA was found better for damage detection than ACO.

Table 6: GA results considering different levels of noise.

\begin{tabular}{ccccccc}
\hline & \multicolumn{2}{c}{$1 \%$ noise } & \multicolumn{2}{c}{$5 \%$ noise } & \multicolumn{2}{c}{$10 \%$ noise } \\
\hline & $N_{\text {elem }}$ & $\alpha$ & $N_{\text {elem }}$ & $\alpha$ & $N_{\text {elem }}$ & $\alpha$ \\
\hline Objective & 230 & 0.2000 & 230 & 0.2000 & 230 & 0.2000 \\
Search 1 & 252 & 0.2290 & 191 & 0.9991 & 191 & 0.9994 \\
Search 2 & 252 & 0.1860 & 191 & 0.9994 & 191 & 0.9996 \\
Search 3 & 252 & 0.1860 & 191 & 0.9989 & 191 & 0.9999 \\
Search 4 & 252 & 0.2290 & 191 & 0.9986 & 191 & 0.9990 \\
Search 5 & 252 & 0.1860 & 191 & 0.9994 & 191 & 0.9990 \\
Mean & 252 & 0.2032 & 191 & 0.9991 & 191 & 0.9994 \\
Deviation & 0 & 0.0235 & 0 & 0.0003 & 0 & 0.0004 \\
Error & $10 \%$ & $2 \%$ & $17 \%$ & $400 \%$ & $17 \%$ & $400 \%$ \\
\hline
\end{tabular}


Table 7: ACO results considering different levels of noise.

\begin{tabular}{ccccccc}
\hline & \multicolumn{2}{c}{$1 \%$ noise } & \multicolumn{2}{c}{$5 \%$ noise } & \multicolumn{2}{c}{$10 \%$ noise } \\
\hline & $N_{\text {elem }}$ & $\alpha$ & $N_{\text {elem }}$ & $\alpha$ & $N_{\text {elem }}$ & $\alpha$ \\
\hline Objective & 230 & 0.2000 & 230 & 0.2000 & 230 & $2.1580 \times 10^{-5}$ \\
Search 1 & 67 & 0.9450 & 191 & 0.9996 & 198 & $2.1610 \times 10^{-5}$ \\
Search 2 & 67 & 0.9449 & 191 & 0.9974 & 198 & $2.0530 \times 10^{-5}$ \\
Search 3 & 67 & 0.9988 & 191 & 0.9860 & 198 & $2.1690 \times 10^{-5}$ \\
Search 4 & 67 & 0.9986 & 191 & 0.9999 & 198 & $7.8280 \times 10^{-5}$ \\
Search 5 & 67 & 0.9829 & 191 & 0.9999 & 198 & $3.2740 \times 10^{-5}$ \\
Mean & 67 & 0.9740 & 191 & 0.9966 & 198 & $2.5460 \times 10^{-5}$ \\
Deviation & 0 & 0.0273 & 0 & 0.0060 & 0 & 0.0004 \\
Error & $71 \%$ & $378 \%$ & $17 \%$ & $398 \%$ & $14 \%$ & $100 \%$ \\
\hline
\end{tabular}

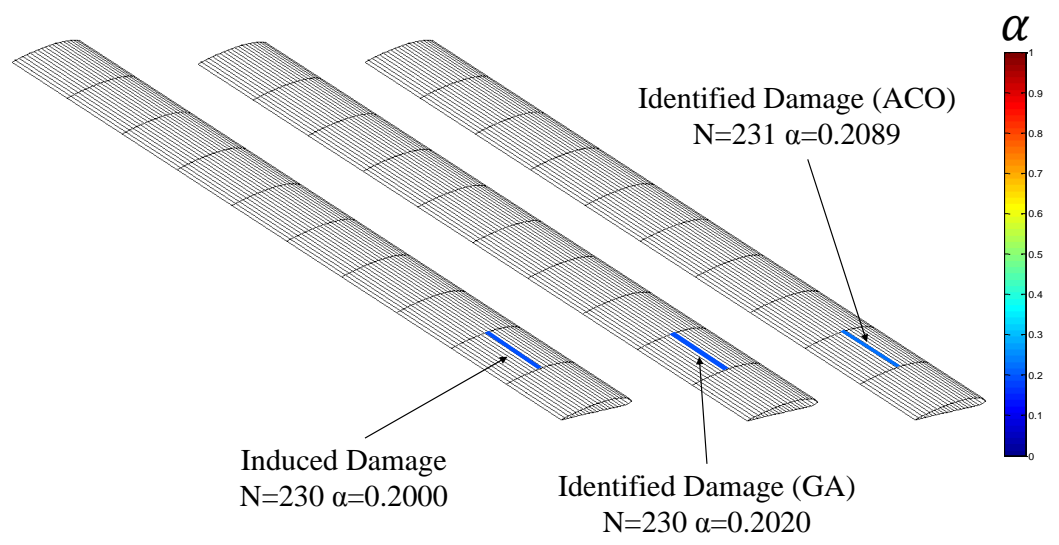

Figure 5 - Damage detection using GA procedure without noisy data.

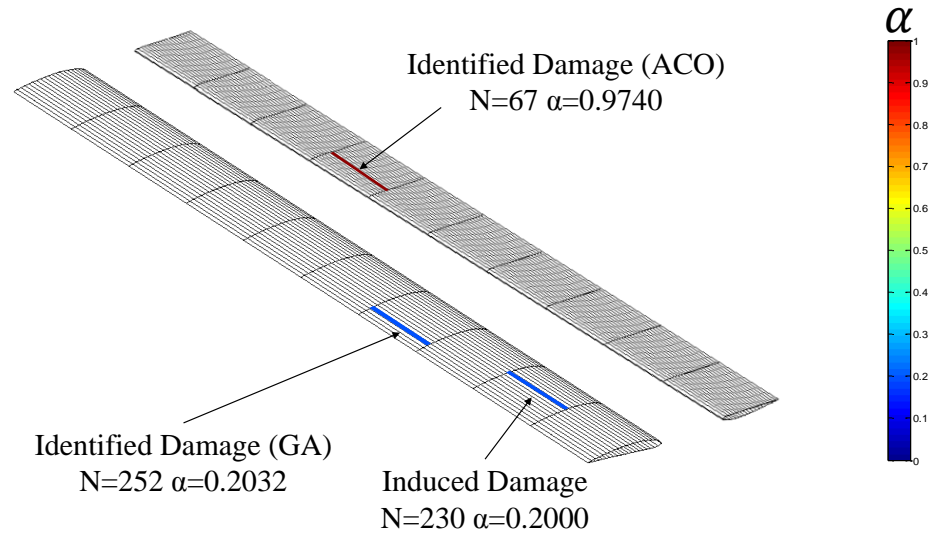

Figure 6 - Damaged laminated plate, detected damages applying the first six natural frequencies after 100 generations for $1 \%$ of GWN. 


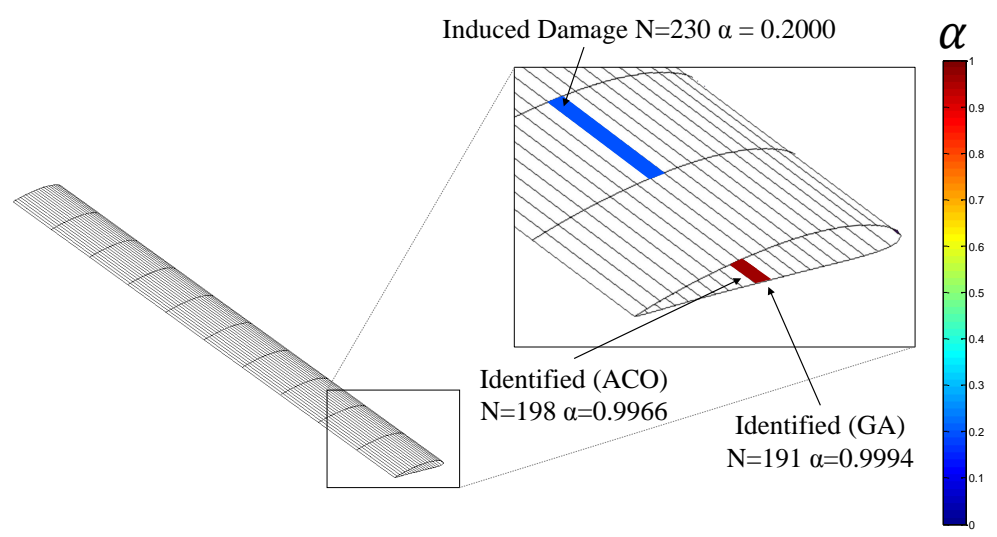

Figure 7 - Damaged laminated plate, detected damages applying the first six natural frequencies after 100 generations for $5 \%$ of GWN.

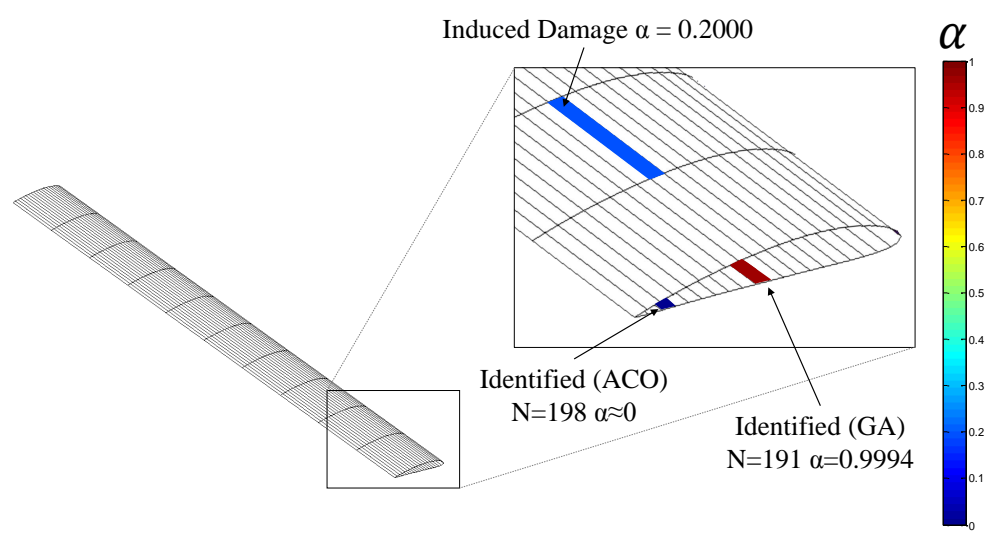

Figure 8 - Damaged laminated plate, detected damages applying the first six natural frequencies after 100 generations for $10 \%$ of GWN.

\section{Conclusions}

In this work, the performance of some well-known modal based damage indexes are tested using GA and ACO. Natural frequencies of a aeronautical profile are employed. Damage is assumed to affect the local stiffness of the shell profile, thus each element stiffness matrix is multiplied by a real number called stiffness factor, having values between 0 and 1 .

The study was focused on the detection and identification of structural damage based on natural frequencies changes of a NACA airfoil of composite material, including finite element numerical modelling and programming for the inverse problem detection using a genetic algorithm.

The results of numerical simulations as well optimization procedures using heuristics methods (GA) as shown a good performance to detect and identify the structural damage modelled as a local stiffness reduction. Normal difficulties appear when noisy data is included in the damage detection process.

This research tested the performance of the GA to identify structural damage, and compared the performance with the ACO. The results generated by GA show significantly less variance among a set of random trials evaluations. Statistical analysis showed that GA yielded better results when compared to ACO, i.e., 
maximum error of $17 \%$ of the damage location considering $10 \%$ of noise. This indicates that better solutions for the same stop criterion.

The evolution of the ability of computational tools of mathematical modelling, numerical simulations beyond the modern computer processing power allow the application and expansion of damage detection method ever more accurate and sophisticated and practical applicability in highly complex structures large number of elements beyond the processing capacity of a vector composed of several design variables in optimization process with use of global heuristics.

Acknowledgments. The authors would like to acknowledge the financial support from the Brazilian agency CAPES (Coordenação de Aperfeiçoamento de Pessoal de Nível Superior) and CNPq (Conhelho Nacional de Desenvolvimento Científico e Tecnológico).

\section{References}

[1] Chen, H.G., Yan, Y.J., Chen, J.S., Wu, Z.Y. Early Damage Detection in Composite Wingbox Structures using Hilbert-Huang Transform and Genetic Algorithm. Structural Health Monitoring, v.6(4), pp.281$298,2007$.

[2] Zhao, X., Qian, T., Mei, G., Kwan, C., Zane, R., Walsh, C., Popovic, Z. Active health monitoring of an aircraft wing with an embedded piezoelectric sensor/actuator network: II. Wireless approaches. Smart Materials and Structures, 16(4), 1218-1225, 2007. http://doi.org/10.1088/0964-1726/16/4/033

[3] Selva, P., Cherrier, O., Budinger, V., Lachaud, F., Morlier, J. Smart monitoring of aeronautical composites plates based on electromechanical impedance measurements and artificial neural networks. Engineering Structures, 56, 794-804, 2013. http://doi.org/10.1016/j.engstruct.2013.05.025

[4] Srinivas, V., Ramanjaneyulu, K., Jeyasehar, C. A. (2011). Multi-stage approach for structural damage identification using modal strain energy and evolutionary optimization techniques. Structural Health Monitoring-an International Journal, 10(2), 219-230. http://doi.org/10.1177/1475921710373291

[5] Abbott, I.H. and Doenhoff A.E.V. Theory of wing sections, including a summary of airfoil data. Courier Corporation, 1959.

[6] Deep, Kusum, Krishna Pratap Singh, M.L. Kansal, and C. Mohan. A real coded genetic algorithm for solving integer and mixed integer optimization problems. Applied Mathematics and Computation, $212(2)$, pp. 505-518, 2009.

[7] Chou, J.-H., Ghaboussi, J. (2001). Genetic algorithm in structural damage detection. Computers Structures, 79(14), 1335-1353. http://doi.org/10.1016/S0045-7949(01)00027-X

[8] Sudha, K. M. and Sukumaran, S. Coherent Genetic Algorithm for Task Scheduling in Cloud Computing Environment. Aust. J. Basic Appl. Sci., 9(2): 1-8, 2015.

[9] Rytter A (1993) Vibration Based Inspection of Civil Engineering Structures, Ph.D Dissertation, Department of Building Technology and Structural Engineering, Aalborg University, Denmark.

[10] Braun, C.E., Chiwiacowsky, L.D., Gomez, A.T. Variations of Ant Colony Optimization for the solution of the structural damage identification problem, Procedia Computer Science, v.51, pp.875-884, 2015. 